\title{
Lesion Sterilization and Tissue Repair-A Recent Novel Approach for the Treatment of Very Uncooperative Pediatric Patients
}

\author{
${ }^{1}$ Teena Gupta, ${ }^{2}$ Gunmeen Sadna, ${ }^{3}$ Neha Aggarwal
}

\begin{abstract}
Introduction: Root canal complexities of deciduous teeth often makes the treatment difficult and makes the child patient uncooperative and sometimes leads to treatment failure. Therefore the noninvasive approach is needed to obtain good cooperation from child patient.
\end{abstract}

Aim: This case report aimed to evaluate the use of triple antibiotic paste clinically-a combination of antibacterial drugs, i.e., metronidazole, minocycline, and ciprofloxacin (3Mix), and propylene glycol as pulp medicament on a necrosed primary molar.

Case description: Two children reported with necrosed primary molar and dentoalveolar abscess were treated with lesion sterilization and tissue repair (LSTR) therapy. The triple antibiotic paste was placed in the access cavity and then sealed with glass-ionomer cement. Subjects were asked for recall visits post-treatment, for clinical and radiographic evaluation.

Conclusion: The study revealed that LSTR treatment showed clinical and radiographic positive response on necrosed primary molars in an uncooperative patient.

Keywords: Dentoalveolar abscess, Endodontic treatment, Lesion STR, Noninstrumentation triple antibiotic paste

How to cite this article: Gupta T, Sadna G, Aggarwal N. Lesion Sterilization and Tissue Repair-A Recent Novel Approach for the Treatment of Very Uncooperative Pediatric Patients. Curr Trends Diagn Treat 2018;2(1):50-53.

Source of support: Nil

Conflict of interest: None

\section{INTRODUCTION}

The major aim of modern dentistry is to maintain and preserve the integrity of the primary dentition until physiologic exfoliation. Loss of primary teeth prematurely can lead to several problems, like eruption sequence disturbance, ectopic eruption and loss of space for succe-

\footnotetext{
${ }^{1}$ Professor, ${ }^{2}$ Head and Professor ${ }^{3}$ Postgraduate Student

${ }^{1-3}$ Department of Pedodontics, Sri Guru Ram Das Institute of Dental Sciences, Amritsar, Punjab, India
}

Corresponding Author: Teena Gupta, Professor, Department of Pedodontics, Sri Guru Ram Das Institute of Dental Sciences, Amritsar, Punjab, India, e-mail: teenasingla@gmail.com daneous permanent teeth. Hence, the preservation of primary tooth structure is necessary, provided that it can be restored to function and remain free from disease. ${ }^{1}$ The pulpectomy treatment in necrotic and abscessed primary teeth shows acceptable results in approximately $85 \%$ of the cases, but it presents various challenges because of the continuous changes in the apical foramen as a result of physiologic and pathologic root resorption. ${ }^{2}$

Endodontic management of necrotic and abscessed primary molars is generally complicated and is more challenging and difficult in uncooperative children due to behavior management problems, restricted mouth opening, root canal system complexities, a risk of damaging the permanent tooth bud and require multiple visits. ${ }^{3}$ Hence keeping in view the need, the Cardiology Research Unit of the Niigata University School of Dentistry developed the concept of LSTR therapy or non-instrumentation endodontic treatment (NIET) which employs the use of triple antibiotic paste for disinfection of oral infectious lesions, which includes dentinal, pulpal, and periapical lesions. ${ }^{4}$

The present study aimed to clinically evaluate LSTR therapy with triple antibiotic paste which includes a mixture of ciprofloxacin, metronidazole, and minocycline (3 Mix-Paste) in endodontic treatment of infected primary teeth, including those with physiologic root resorption.

\section{CASE DESCRIPTION}

The clinical procedure of LSTR and final restoration performed on each of the two cases are presented here. After complete dental examination with appropriate radiographs, a clinical diagnosis was made. Informed consent was obtained prior to the treatment after discussion of the risks, benefits, and alternatives with the parents.

\section{Preparation of 3-mix Paste}

The chemotherapeutic agents used, ciprofloxacin, metronidazole, and minocycline, were pulverized into powder after the removal of the enteric coating on the drugs and were kept separately in tightly capped containers to prevent exposure to moisture and light. These powdered drugs were then mixed in a ratio of 1:3:3 with propylene glycol to form 3-mix paste. 


\section{Case 1}

A 6-year-old male child referred by a local dental practitioner reported to the Department of Pedodontics, SGRD Amritsar with the chief complaint of pain in the lower left back tooth region. The patient was highly, and the parents gave a history of intermittent pain for the past two weeks, with the intensity of pain increasing especially during the nights. On clinical examination, the dentoalveolar abscess was seen in the lower left first primary molar. The intraoral periapical radiograph showed periapical radiolucency circumscribing the distal root of the first primary molar (Fig. 1A). Because of the uncooperative and anxious behavior of the child, a systemic antibiotic
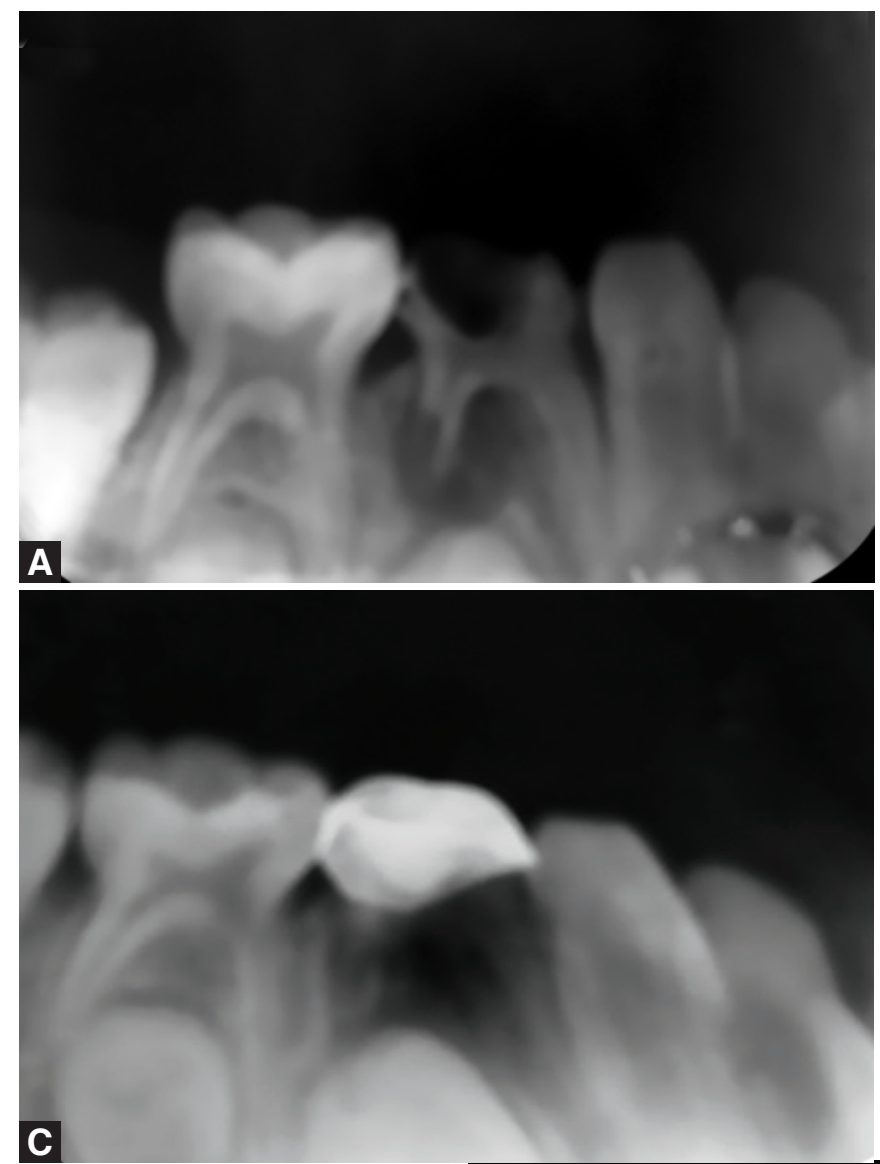

was prescribed and NIET using the triple antibiotic paste was planned for disinfection of the pulp and periapical lesion. The access opening was done in the primary first molar, and the coronal pulp tissue was extirpated under local anesthesia. Then the triple antibiotic paste was placed in the pulp chamber over the root canal orifices, and glass ionomer restoration was given as a coronal seal followed by a stainless steel crown (Fig. 1B). At onemonth follow-up, the patient was asymptomatic with the absence of pain, and after 3 and 6 months the radiograph showed a definite reduction in periapical radiolucency with bone formation in the periradicular region (Figs $1 \mathrm{C}$ to $\mathrm{E})$.
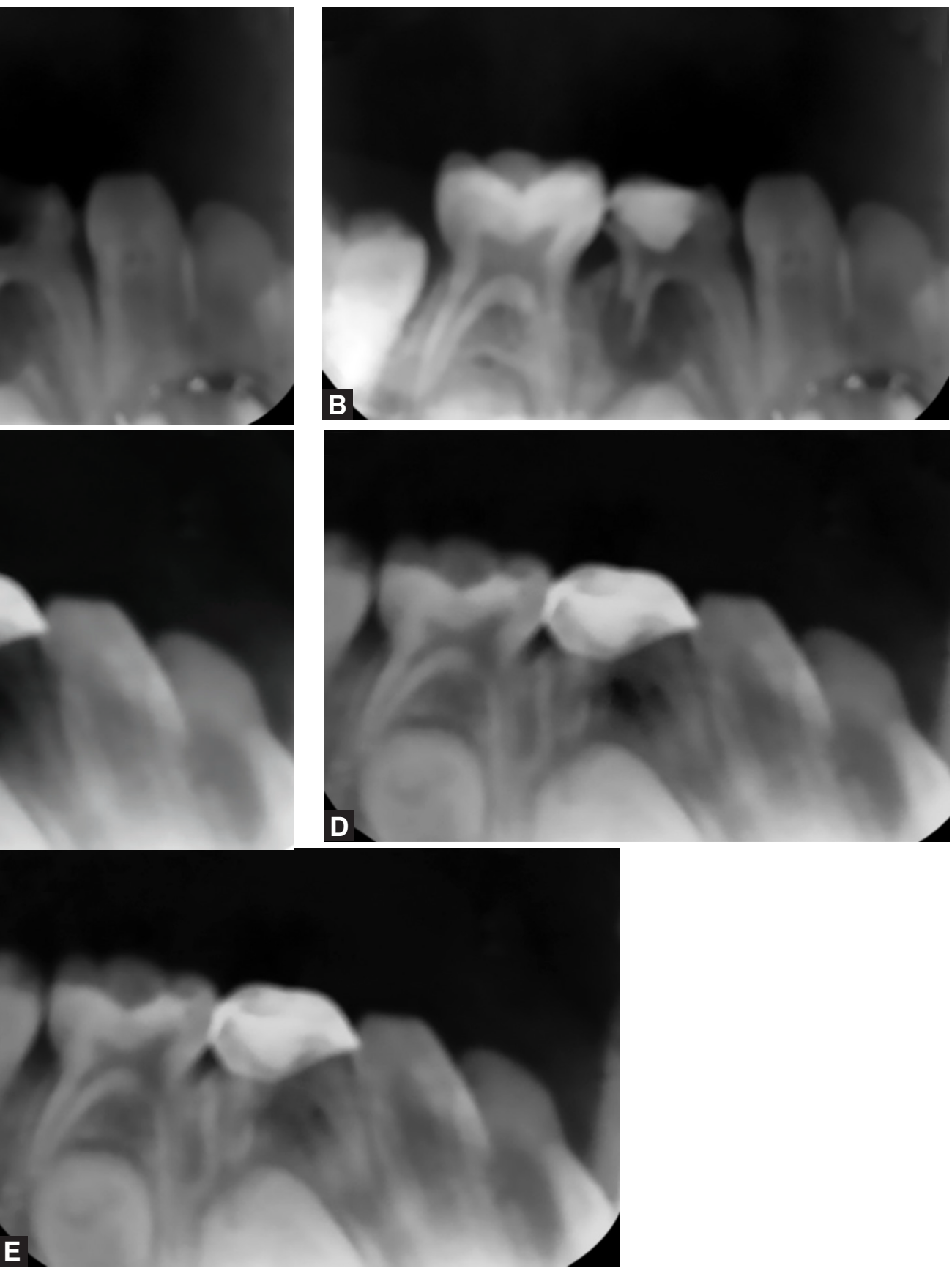

Figs $1 \mathrm{~A}$ to E: Pre- and postoperative periapical radiograph of lower left first deciduous molar after LSTR. at 1,3 and 6 months follow-up 


\section{Case 2}

The second case describes the endodontic treatment done on anxious six and a half-year-old male child reported to the Department of Pedodontics, with the chief complaint of pain and swelling in the lower left back tooth region from past two days. On clinical examination, the dentoalveolar abscess was seen in left lower back tooth region with proximally carious second molar and grossly carious Grade 2 mobile first primary molar. The periapical radiograph showed resorption of roots in the first molar and periradicular radiolucency with resorption of the distal root in the second molar region along with furcation involvement (Fig. 2A). Systemic antibiotics and analgesics were prescribed. Lesion sterilization therapy for second molar and extraction of the first molar followed by crown and loop space maintainer was planned. After the treatment, follow-ups were done at 1, 6 and 12 months (Fig. 2B). At 6-month follow up, the patient was asymptomatic with the absence of pain (Fig. 2C). After one year the periapical radiograph showed healing of periapical lesion with an increase in mineral density in bone and reduction in periapical radiolucency (Fig. 2D).

\section{DISCUSSION}

The results demonstrate that LSTR treated primary teeth with periradicular lesions and root resorption gave excel- lent clinical results, even in a single visit. LSTR therapy aims to eliminate the bacteria from the root canals and does not depends on mechanical procedures. Therefore the clinical procedure is simple and does not require long chair-time or multiple visits. Metroni-dazolehas a wide spectrum of bactericidal action against oral obligate anaerobes. However, some bacteria in lesions are resistant to metronidazole indicating the necessity of additional antibacterial drugs to sterilize these lesions, and thus ciprofloxacin and minocycline were added to eliminate all the bacteria. ${ }^{1}$ Sato et al. first formulated the use of Metronidazole (500 mg), ciprofloxacin (200 mg) and Minocycline (100 mg) with a suitable carrier to enhance effective destruction of endodontic pathogens (aerobic as well as anaerobic). This combination has been popularly known as 3-mix paste/ triple antibiotic paste. ${ }^{5}$ The penetration ability of these drugs to sterilize deep layers of infected root dentine was improved by mixing these drugs with propylene glycol to form the ointment base. ${ }^{6}$

In the advent of internal/external root resorption or physiologic root resorption exceeding $2 / 3$ rd root length that requires short-term space management, LSTR with 3Mix-Paste may be considered as an effective alternative procedure. ${ }^{7}$ This technique may also find application
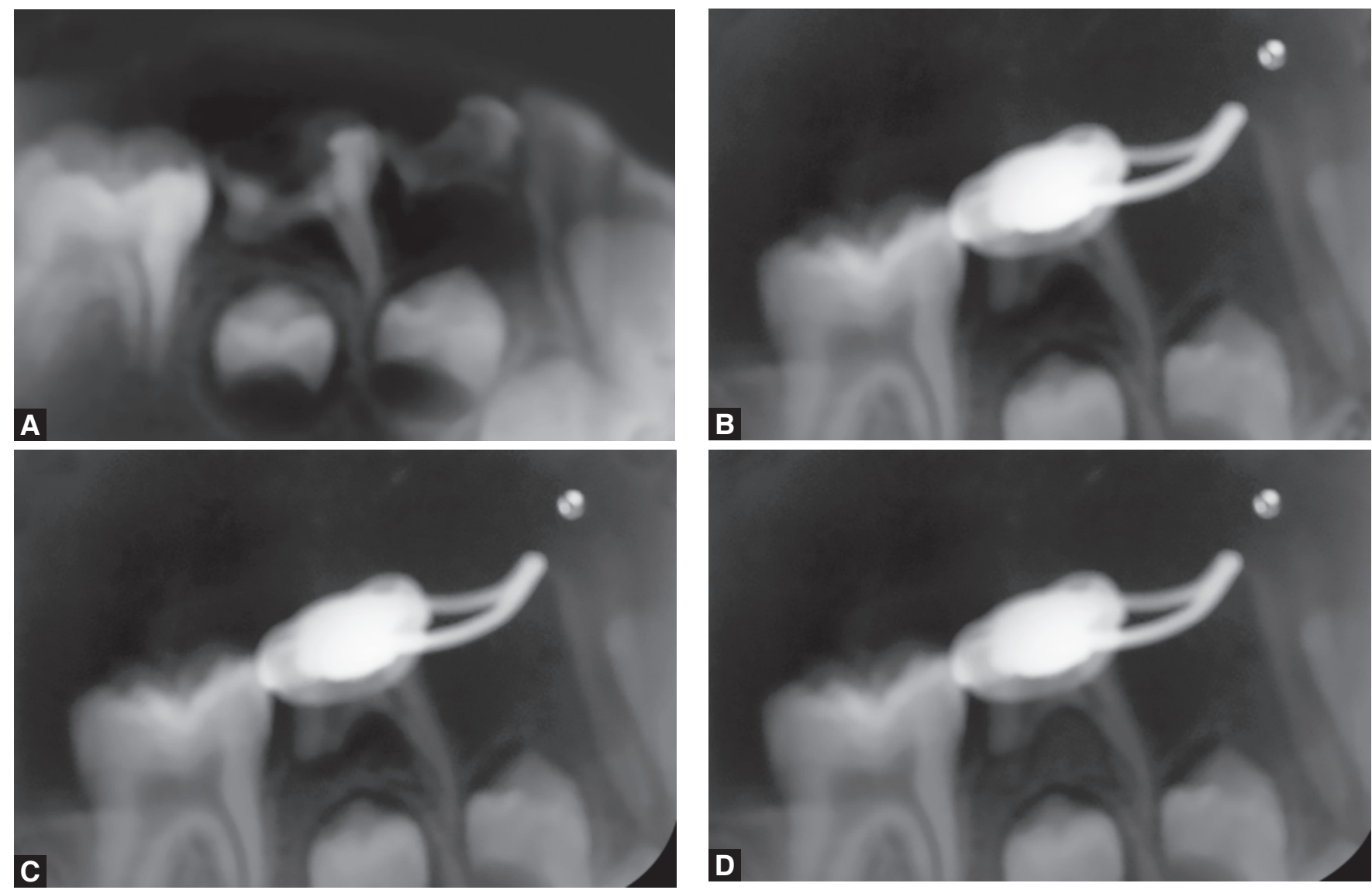

Figs 2A to D: Pre- and postoperative periapical radiograph of a lower left second deciduous molar with crown and loop space maintainer at 1, 6 and 12 follow-up 
in children with special needs in whom conventional endodontic treatment cannot be performed due to associated medical conditions. It may also find application in managing pulpal infections in uncooperative patients in a single visit. This application of LSTR technique has been researched and reported to be successful. ${ }^{8}$

One of the risks associated with the local application of antibiotics is the discoloration of tooth and hypersensitivity reaction. However, in our study, none of the cases showed any evidence of such a reaction to the antibiotic combination. Though the application of small volumes of the drugs showed no side effects, care should be taken if children are sensitive to chemicals or antibiotics.

\section{CONCLUSION}

Within the limitations of this study, LSTR therapy using 3-mix paste, i.e., a mixture of ciprofloxacin, metronidazole, minocycline (3-mix), and propylene glycol, proved as a successful approach in treating the infected root canals of primary teeth in uncooperative patients.

\section{CLINICAL SIGNIFICANCE}

The LSTR single sitting technique shows good clinical and radiographic success rates in the endodontic treatment of very uncooperative patients.

\section{REFERENCES}

1. Jaya AR, Praveen P, Anantharaj A, Venkataraghavan K, Rani PS. In vivo evaluation of lesion sterilization and tissue repair in primary teeth pulp therapy using two antibiotic drug combinations.J ClinPediatr Dent. 2012 Winter;37(2):189-191.

2. Burrus D, Barbeau L, Hodgson B. Treatment of abscessed primary molars utilizing lesion sterilization and tissue repair: literature review and report of three cases.Pediatr Dent. 2014 May-Jun;36(3):240-244.

3. Trairatvorakul C, Detsomboonrat P. Success rates of a mixture of ciprofloxacin, metronidazole, and minocycline antibiotics used in the non-instrumentation endodontic treatment of mandibular primary molars with carious pulpal involvement. Int J Paediatr Dent. 2012 May;22(3):217-227.

4. Takushige T, Cruz Ev, Moral A, Hoshino E. Endodontic Treatment of primary teeth using combination of antibacterial drugs. IntEndod J. 2004;37:132-138.

5. Sato I, Ando-Kurihara N, Kota K, et al. Sterilization of infected root-canal dentine by topical application of a mixture of ciprofloxacin, metronidazole and minocycline in situ. IntEndod J. 1996;29(2):118-124.

6. Cruz EV, Kota K, Huque J, Iwaku M, Hoshino E. Penetration of propylene glycol into dentine. IntEndod Journal. 2002;35:330336.

7. Rocha MJ, Cardoso M, Endodontic treatment of traumatized primary teeth - Part2. Dent. Traumatol. 2004;20(6):314-326.

8. Hoshino E, Asgor MA, Yagi M, Garcia MCG, Cruz EV, Oyanagi $\mathrm{H}$, Kota K. Oral Health program using LSTR 3 Mix- MP NIET Therapy: Pulp Cell Behaviour: Pulp Therapy and Endod. 2006;1786. 